\title{
Ivermectina para Demodex y condiciones oculares asociadas: revisión de la literatura
}

\section{Ivermectin for Demodex and related ocular conditions: Review of the literature}

\author{
Juan G. Gaviria-Carvajal ${ }^{1 *}$, Carolina Torres-Triviño ${ }^{2}$, Iván F. Suárez-Franco², Paolo Solano Leal ${ }^{1}$ y Maura Cediel ${ }^{1}$
}

${ }^{1}$ Nazar Grupo Oftalmológico, Ibagué, Tolima; ${ }^{2}$ Salud y Láser, Bogotá. Colombia

\begin{abstract}
Resumen
Introducción: Existe creciente interés en Demodex y su asociación con condiciones como rosácea, blefaritis, chalazión, meibomitis y queratoconjuntivitis. El manejo con ivermectina ha sido ampliamente reportado tanto en dermatología como en oftalmología. Objetivo: Revisar la literatura para determinar el papel de Demodex en la inflamación de la superficie ocular y el uso de ivermectina en su tratamiento. Diseño del estudio: Revisión de la literatura. Método: Búsqueda de artículos en PubMed con los siguientes términos: Demodex, rosácea ocular, chalazión, rosácea pediátrica, meibomitis, queratoconjuntivitis e ivermectina. Resultados: Se presenta una revisión de generalidades, epidemiología y fisiopatología de la inflamación ocular asociada a Demodex. Se resume el conocimiento actual sobre rosácea y Demodex, y el papel de este como disparador de procesos inflamatorios como chalazión, blefaritis, meibomitis, queratoconjuntivitis y rosácea ocular. Se citan estudios relevantes sobre el manejo de ivermectina en las condiciones mencionadas. Conclusiones: La evidencia reciente otorga a Demodex el papel de un agente disparador de varias condiciones inflamatorias de la superficie ocular. Si bien se requieren más estudios para determinar la efectividad de la ivermectina tópica, el conocimiento actual permite pensar que puede ser útil contra Demodex por su capacidad acaricida.
\end{abstract}

Palabras clave: Demodex. Rosácea ocular. Chalazión. Meibomitis. Queratoconjuntivitis. Ivermectina.

\section{Abstract}

Background: There has been increasing interest in Demodex and its association with conditions such as rosacea, blepharitis, chalazion, meibomitis and keratoconjunctivitis; and ivermectin as a treatment has been reported both in dermatology and ophthalmology. Objective: To review the literature in order to determine the role of Demodex in ocular surface inflammation and the use of ivermectin for its treatment. Study design: Review of the literature. Methods: An article search was done in PubMed with the following terms: Demodex, ocular rosacea, chalazion, pediatric rosacea, meibomitis, keratoconjunctivitis and ivermectin. Results: A review including the epidemiology and pathophysiology of ocular inflammation associated with Demodex is presented. Current knowledge on Demodex and rosacea, chalazion, blepharitis, meibomitis, keratoconjunctivitis and ocular rosacea is summarized. Relevant articles on the use of ivermectin for these conditions are listed. Conclusions: Recent evidence suggests Demodex is a trigger for a number of ocular surface inflammatory conditions. Although more studies are necessary to determine the effectiveness of topical ivermectin, current knowledge supports its acaricidal action against Demodex.

Key words: Demodex. Ocular rosacea. Chalazion. Meibomitis. Keratoconjunctivitis. Ivermectin.

Correspondencia:

*Juan G. Gaviria-Carvajal

E-mail: drjuangaviria@gmail.com

CC BY-NC-ND (http://creativecommons.org/licenses/by-nc-nd/4.0/).
Fecha de recepción: 18-11-2020

Fecha de aceptación: 30-05-2021

DOI: 10.24875/RSCO.M21000013
Disponible en internet: 28-12-2021 Rev Soc Colomb Oftalmol. 2021;54(2):86-91

www.revistaSCO.com 


\section{Generalidades sobre Demodex}

Demodex es un ácaro que convive en la unión pilosebácea y hace parte de la microflora de la piel facial. Se reconocen dos especies: folliculorum y brevis ${ }^{1}$.

En biopsias de piel se encuentran Demodex en un $10 \%$ de la población, y de los dos, $D$. folliculorum es el más común, permanece en grupos de 10-15 individuos y se encuentra especialmente en los folículos de las pestañas, la frente, región malar, pliegues nasolabiales y nariz. $D$. brevis es solitario y se encuentra en canales auriculares, tronco, pubis y glándulas de Meibomio. En general, los hombres tienen mayores índices de infestación, pues los andrógenos estimulan la producción sebácea.

\section{Epidemiología}

Un estudio en población pediátrica encontró Demodex en las pestañas en el $12 \%$ de 1,575 pacientes entre los 3 y 14 años ${ }^{2}$.

La incidencia aumenta con la proliferación de las glándulas sebáceas durante la pubertad y sigue aumentando con los años, llegando a ser del $95 \%$ en personas mayores de 71 años. En un estudio realizado en Colombia, la prevalencia de Demodex es del $42.1 \%$ y alcanza el $63.2 \%$ en pacientes con blefaritis ${ }^{2}$. El aumento de la incidencia de blefaritis con la edad se correlaciona con la expansión de la población de ácaros.

Los pacientes afectados por VIH pueden presentar demodicosis clínica, con una erupción que genera prurito en la cara cuando el conteo de CD4 es menor de $200 / \mathrm{mm}^{3}$. Este tipo de erupciones usualmente responde al tratamiento con ivermectina oral ${ }^{3}$. También se ha reportado infestación por Demodex en pacientes con linfoma o leucemia, así como una mayor densidad de Demodex en diabéticos.

\section{Fisiopatología y respuesta inmunitaria ante Demodex}

La infestación por Demodex aumenta la presencia de bacterias como estreptococos, estafilococos $y$ Bacillus oleronius, y causa reacciones de hipersensibilidad. Los ácaros producen microabrasiones que inducen hiperplasia epitelial e hiperqueratinización reactiva. Las proteínas y detritos del ácaro pueden disparar respuestas inflamatorias de tipo hipersensibilidad por un incremento en el número de células $T$ CD4, macrófagos y células de Langerhans. Aunque muchos pacientes no presentan una inflamación significativa, existe evidencia de que Demodex genera una respuesta inflamatoria fuerte. En secciones histológicas se observan espongiosis e infiltrados linfocitarios alrededor de los folículos infestados, pero no alrededor de los folículos no infestados ${ }^{4}$. $D$. brevis se aloja en las glándulas de Meibomio; y su exoesqueleto de quitina puede causar una reacción granulomatosa a cuerpo extraño que genera obstrucción.

Al evaluar pacientes con rosácea y Demodex mediante reacción en cadena de la polimerasa, se ha encontrado un incremento de citocinas proinflamatorias como el factor de necrosis tumoral alfa, interleucina (IL) $1 \mathrm{~b}$ e IL-8, especialmente en la variedad papulopustular. También se ha encontrado una mayor cantidad de citocinas proinflamatorias en la lágrima de pacientes con blefaritis asociada a Demodex en comparación con un grupo con blefaritis sin Demodex y en un grupo de controles sanos ${ }^{5}$.

\section{Condiciones dermatológicas asociadas a Demodex}

La presencia de tapones foliculares causados por la protrusión de ácaros y queratina a partir del folículo se denomina pitiriasis folliculorum. La presencia de grandes cantidades de ácaros puede aparecer como una papila folicular y se correlaciona con prurito. $D$. brevis tiende a producir una erupción papulopustular en distribución malar sobre la piel enferma. $D$. folliculorum se ha visto más asociado a eritema y descamación en la frente y la nariz. Demodex causa erupciones inflamatorias crónicas que semejan foliculitis bacterianas, rosácea y dermatitis perioral, y se ha reportado la alopecia por Demodex, similar a la sarna de los animales.

\section{Rosácea y Demodex}

La rosácea es una enfermedad inflamatoria de la piel que afecta principalmente mejillas, nariz, mentón y frente. Clásicamente, se clasifica en cuatro subtipos: eritemato-telangiectásica, pápulo-pustulosa, fimatosa y ocular. Varios estudios sobre fisiopatología de la rosácea han mostrado aumento de la densidad de Demodex, donde se activa una respuesta inmunitaria por la vía de los receptores tipo toll 2 (TLR-2), generando un aumento de mediadores proinflamatorios y las lesiones en piel características ${ }^{6,7}$. Los signos y síntomas de la rosácea papulopustular se correlacionan con la frecuencia de infestación por Demodex; y la densidad de 
Demodex (número del Demodex por unidad de área) se correlaciona con la severidad de la enfermedad ${ }^{8}$.

\section{Condiciones oftalmológicas asociadas a Demodex}

Coston describió por primera vez la blefaritis por Demodex ${ }^{9}$ y desde entonces se ha asociado a otras condiciones oculares ${ }^{10} . D$. folliculorum produce agrandamiento e hiperqueratinización del folículo con formación de caspa cilíndrica, pérdida de pestañas, obstrucción y disfunción de glándulas de Meibomio. La blefaritis anterior se asocia con $D$. folliculorum y afecta a la población mayor, mientras que la blefaritis posterior con disfunción de glándulas de Meibomio se asocia a $D$. brevis y se observa en pacientes más jóvenes. Uno de los síntomas más característicos de blefaritis por Demodex es el prurito y no es inusual que los pacientes afectados refieran la sensación de «animales caminando". Muchos pacientes refieren ardor, sensación de cuerpo extraño, enrojecimiento y molestias inespecíficas y vienen siendo tratados por años como síndrome de ojo seco, y se ha encontrado que entre los pacientes con diagnóstico de ojo seco, aquellos que tienen Demodex presentan puntuaciones más altas en el cuestionario OSDI (Ocular Surface Disease Index $)^{11}$.

A la biomicroscopia, la caspa cilíndrica, un hallazgo patognomónico, se ve mejor en aumentos de 10 y $16 \mathrm{X}$ (Fig. 1), mientras que la observación de las colas de ácaros en la base de pestañas en 25 o 40X permite un diagnóstico simple y directo (Fig. 2).

La microscopia de pestañas consiste en retirar dos o tres pestañas de cada párpado, y colocarlas sobre una lámina portaobjetos para llevarlas a visualización en un microscopio de luz. Se puede añadir fluoresceína para resaltar el exoesqueleto. Es posible registrar imágenes y vídeos del parásito en movimiento con aumentos de 10 a $60 \mathrm{X}$, que causan gran impacto en el paciente cuando se quiera mejorar la adherencia al tratamiento ${ }^{12}$

\section{Chalazión}

Los chalazión causan dolor, inflamación y alteración estética. Con frecuencia son recurrentes y motivo de preocupación. Estudios recientes confirman que $D$. folliculorum ${ }^{13}$ y $D$. brevis ${ }^{14}$ pueden ser causantes de chalazión de repetición en pacientes adultos y en población pediátrica.

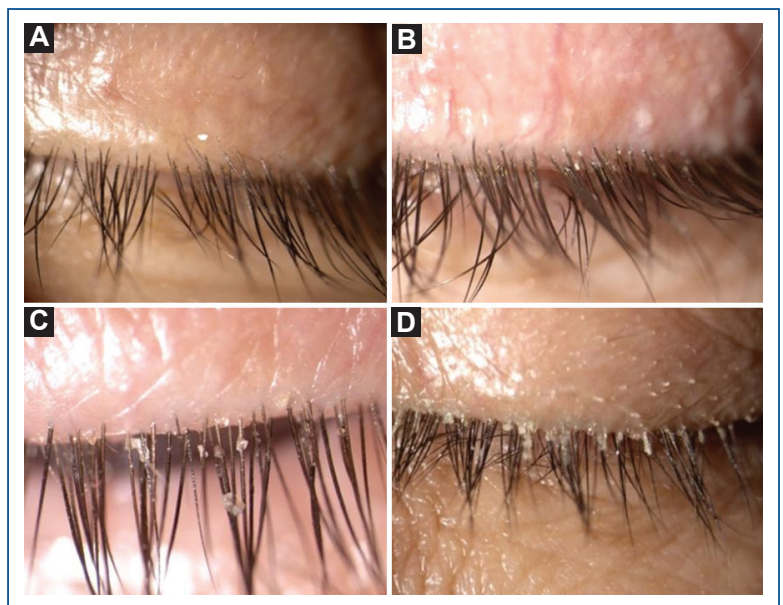

Figura 1. Clasificación de caspa cilíndrica. Grado 0 (A): no se observa. Grado 1 (B): caspa cilíndrica solo en un sector del párpado. Grado 2 (C): moderada en ambos párpados. Grado 3 (D): abundante en ambos párpados (adaptada de Elston, et al., 2014').

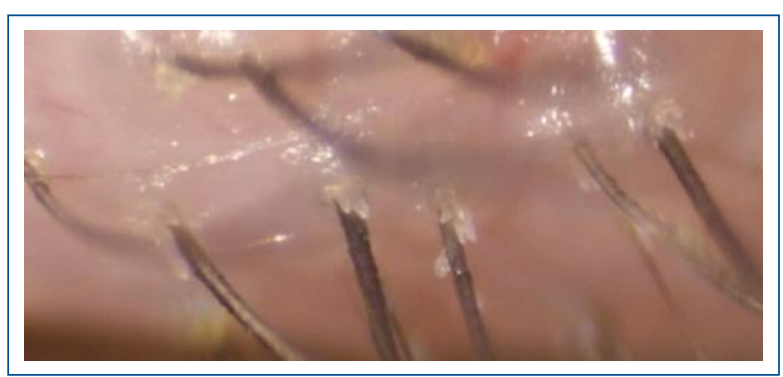

Figura 2. Visualización directa de Demodex en lámpara de hendidura: con enfoque en la base de las pestañas en 25X, se observan grupos de 3-4 colas de Demodex en cada pestaña. Este hallazgo es confirmatorio de infestación por Demodex.

\section{Disfunción de glándulas de Meibomio, meibomitis y queratoconjuntivitis}

En 2019, Cheng y colaboradores, en un estudio de casos y controles, encontraron una tasa de infección por Demodex del $89.32 \%$ con cambios significativos en la anatomía glandular evaluados mediante microscopia confocal ${ }^{15}$. Reportes anteriores encuentran asociación entre $D$. brevis y lesiones corneales ${ }^{16}$ bilaterales en dos tercios de los pacientes, que se acompañan de blefaritis en el $90 \%$ de los casos. Los signos incluyen infiltración corneal marginal con neovascularización, queratitis punteada superficial, opacidad corneal superficial, lesiones flictenuloides e incluso perforación corneal (Fig. 3) $)^{17,18}$. 


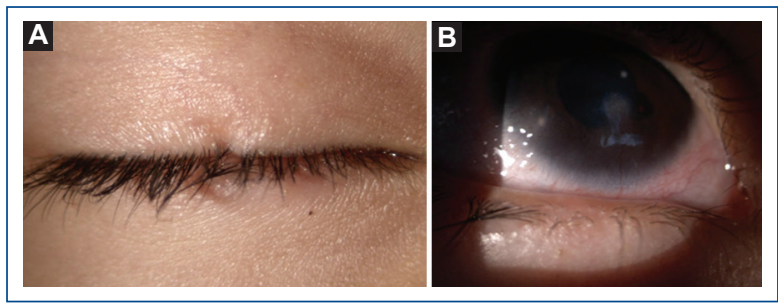

Figura 3. Blefaroqueratoconjuntivitis crónica.

A: irregularidad del margen palpebral como secuela de blefaritis y chalazión de repetición en una niña de cuatro años. B: neovascularización corneal inferior con cicatrices de queratitis marginal y paracentral que afectan la agudeza visual.

Estos hallazgos pueden confundirse con los de queratitis herpética o conjuntivitis alérgicas durante años, llevando con frecuencia a diagnósticos y tratamientos no efectivos. Liang, et al. encontraron que esta queratitis es severa y común entre los pacientes jóvenes afectados por Demodex, con una correlación importante entre el grado de la queratitis y la alteración de las glándulas de Meibomio. Después de realizar un análisis de regresión multivariante, los autores encontraron dos factores que hacían peor la severidad de la queratitis: meibomitis del párpado superior y confirmación de $D$. brevis ${ }^{19}$. Según lo anterior, Demodex se añade al listado de gérmenes capaces de producir alteraciones de glándulas de Meibomio y queratoconjuntivitis, como son Staphylococcus epidermidis, Staphylococcus aureus ${ }^{20}$ y bacterias que pueden inducen hipersensibilidad retardada, como Propionibacterium acnes ${ }^{21}$.

\section{Rosácea ocular pediátrica}

La rosácea ocular en niños presenta un reto diagnóstico porque puede confundirse con otras patologías y los hallazgos cutáneos no siempre están presentes. En sus inicios, estos pacientes pueden presentar orzuelos y chalazión de repetición. La enfermedad es más frecuente en niñas y comúnmente asimétrica (Fig. 4). Sin diagnóstico ni tratamiento, estos pacientes desarrollarán blefaroqueratoconjuntivitis crónica y pueden presentar neovascularización corneal (Fig. 4) y lesiones flictenulares que pueden llevar a pérdida visual. Los hallazgos corneales en pacientes con Demodex de Kheirkhah, Liang y, más recientemente, Patel $^{22}$, son prácticamente los mismos de la queratoconjuntivitis asociada a meibomitis descrita por Suzuki ${ }^{23}$ y la queratoconjuntivitis meibomiana descrita por McCulley ${ }^{23}$, y coinciden con la clínica de la rosácea

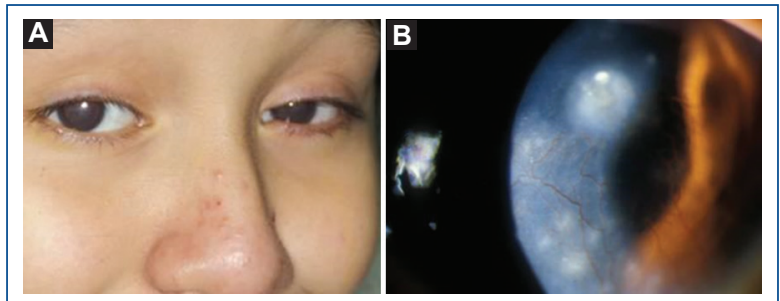

Figura 4. Rosácea ocular pediátrica. A: rosácea ocular pediátrica en una paciente de siete años que presentaba pápulas y pústulas a nivel del dorso nasal y compromiso palpebral. A nivel corneal (B) presenta lesiones de aspecto flictenular, neovascularización corneal extensa, opacidad y queratitis de predominio inferior, con alteración de la agudeza visual.

ocular pediátrica ${ }^{24}$, por lo cual, hasta que no se realicen estudios adicionales, no podemos descartar que se trate de la misma condición, aunque cada autor utilice el término que considere más apropiado.

\section{Rosácea ocular}

Las características diagnósticas mayores de la rosácea cutánea según la clasificación de $2017^{25}$ incluyen eritema centrofacial y cambios fimatosos, flushing, pápulo-pústulas y telangiectasias. Estos hallazgos pueden equipararse a nivel ocular con eritema palpebral, hiperemia conjuntival, chalazión de repetición y compromiso obstructivo de las glándulas de meibomio, telangiectasias del margen palpebral y neovascularización corneal, respectivamente. Las características secundarias son: ardor o prurito, piel seca y edema, que, a su vez, pueden equipararse en la rosácea ocular con sensación de cuerpo extraño, prurito y fotofobia, ojo seco y engrosamiento e irregularidad del margen palpebral, respectivamente. Podemos entonces ver cómo la rosácea ocular y la cutánea comparten características clínicas que varían solo en su localización anatómica, y que los síntomas dependen del lugar afectado. Si bien no está clara la asociación entre rosácea cutánea y Demodex, algunos autores consideran que comparten características comunes y podrían considerarse la misma enfermedad con presentaciones diferentes ${ }^{26}$.

\section{Tratamientos para Demodex}

No todo paciente con Demodex requiere tratamiento, pues ha sido considerado por muchos un parásito normal de la piel humana. Favorecemos el tratamiento en 
pacientes mayores sintomáticos y cuando buscamos prevenir complicaciones futuras, especialmente en niños y jóvenes con rosácea, blefaroqueratoconjuntivitis, meibomitis o chalazión de repetición.

Tanto la exfoliación mecánica de párpados como la higiene en casa reducen la población de ácaros. Se favorece el uso de limpiadores diseñados para la piel de los párpados, pues el champú para bebés puede afectar los niveles de MUC5AC y la población de células caliciformes. Se ha reportado el uso de ivermectina y metronidazol oral y tratamientos tópicos ya en desuso como el óxido de mercurio y el gel de sulfuro de metronidazol. La pilocarpina en gel al $4 \%$ y el metronidazol tópico al $2 \%$ han demostrado reducción del conteo de Demodex. La permetrina en crema al $5 \%$ es un insecticida para el tratamiento de la escabiosis y ha demostrado ser superior al gel de metronidazol al $0.75 \%$. El aceite de árbol de té en concentraciones superiores al $5 \%$ ha demostrado ser acaricida.

\section{Ivermectina}

La ivermectina es un antiparasitario ampliamente usado contra parasitosis como filariasis, oncocerca, ascaridiasis y escabiosis que actúa al aumentar la permeabilidad de canales de cloro en el sistema nervioso periférico, causando parálisis y eventual muerte de ácaros y parásitos ${ }^{27}$. Se le atribuyen también propiedades antiinflamatorias, pues disminuye la expresión del TLR-2, la catelicidina (IL-37), la calicreína 5 (KLK-5) y otras vías proinflamatorias relacionadas con la fisiopatología de la rosácea.

\section{Ivermectina para Demodex y rosácea}

Se ha usado con éxito por vía oral en casos de rosácea papulopustular severa que no responden a diversos tratamiento ${ }^{28}$ y por vía tópica para rosácea papulopustular leve a moderada gracias a su efecto antiinflamatorio y acaricida. Ha demostrado mejor respuesta clínica y tolerancia que otras terapias tópicas como el metronidazol o el ácido azelaico; por lo cual se ha incluido recientemente en guías de manejo de rosácea cutánea ${ }^{29-31}$. Sobolewska, et al. recientemente han reportado resultados favorables en rosácea ocular con ivermectina al $1 \%$ en un estudio piloto de 10 pacientes $^{32}$.

\section{IVERMECTINA Y BLEFARITIS}

El tratamiento tópico realizando una limpieza con aceite de árbol de té seguido por masajes, disminuye efectivamente la población de ácaros y la inflamación ocular. En un estudio con ivermectina oral, se redujo el número de $D$. folliculorum y se mejoró el tiempo de ruptura de la lágrima. Recientemente, Ávila, et al..$^{33}$ reportaron una erradicación del $96.6 \%$ de Demodex usando un gel con ivermectina al $0.1 \%$ y metronidazol al $1 \%$.

\section{Discusión}

Tradicionalmente hemos pensado en la demodicosis como una condición inofensiva causada por un parásito comensal saprófito. Sin embargo, numerosos estudios confirman una fuerte asociación entre la actividad de la rosácea cutánea y la presencia y población de Demodex. En oftalmología, la evidencia también demuestra que Demodex puede ser patogénico en algunos pacientes; por ejemplo, en blefaritis anteriores sintomáticas con una elevada población de ácaros o en pacientes jóvenes que presentan signos de rosácea ocular como meibomitis, chalazión de repetición y queratoconjuntivitis.

Las estrategias para el manejo de la blefaritis por Demodex son variadas e incluyen la higiene palpebral y antibióticos tópicos o sistémicos. El uso de ivermectina oral o en crema ha sido incluido en las guías de manejo para el de la rosácea eritemato-telangiectásica y algunos estudios en oftalmología respaldan su efectividad para blefaritis y rosácea ocular.

\section{Conclusión}

Se requiere un alto índice de sospecha y un examen detallado para diagnosticar rosácea ocular en niños, meibomitis y demodicosis. Una vez identificada la presencia de Demodex en el escenario de las anteriores patologías, tiene sentido realizar tratamiento para evitar futuras complicaciones y reducir recurrencias. Se requieren más estudios para determinar la efectividad de la ivermectina tópica, aunque el conocimiento actual indica que puede ser efectiva en el tratamiento de Demodex y condiciones asociadas.

\section{Financiamiento}

La presente investigación no ha recibido ninguna beca específica de agencias de los sectores públicos, comercial, o con ánimo de lucro.

\section{Conflicto de intereses}

El Dr. Juan G. Gaviria es asesor científico de laboratorios I-LAB. Los demás autores no reportan conflicto de intereses relacionados con el presente trabajo. 


\section{Responsabilidades éticas}

Protección de personas y animales. Los autores declaran que para esta investigación no se han realizado experimentos en seres humanos ni en animales.

Confidencialidad de los datos. Los autores declaran que han seguido los protocolos de su centro de trabajo sobre la publicación de datos de pacientes.

Derecho a la privacidad y consentimiento informado. Los autores han obtenido el consentimiento informado de los pacientes y/o sujetos referidos en el artículo. Este documento obra en poder del autor de correspondencia.

\section{Bibliografía}

1. Elston CA, Elston DM. Demodex mites. Clin Dermatol. 2014;32:739-43.

2. Galvis-Ramírez L, Álvarez-Osorio JJ, Rey-Serrano. Prevalencia de infección por Demodex folliculorum en pacientes que acuden a consulta general de oftalmología. Rev Salud Publica. 2011;13:990-7.

3. da Rocha MC, Travassos AR, Uva L, Sequeira H, Filipe P. Demodicosis treatment with systemic ivermectin. Skinmed. 2017:15:293-5.

4. Casas C, Paul C, Lahfa M, Livideanu B, Lejeune O, Alvarez-Georges S, et al. Quantification of Demodex folliculorum by PCR in rosacea and its relationship to skin innate immune activation. Exp Dermatol. 2012;21(12):906-10.

5. Kim JT, Lee SH, Chun YS, Kim JC. Tear cytokines and chemokines in patients with Demodex blepharitis. Cytokine. 2011;53:94-9.

6. Forton FMN, de Maertelaer V. Two consecutive standardized skin surface biopsies: an improved sampling method to evaluate Demodex density as a diagnostic tool for rosacea and demodicosis. Acta Derm Venereol. 2017;97:242-8.

7. Holmes $A D$, Steinhoff M. Integrative concepts of rosacea pathophysiology, clinical presentation and new therapeutics. Exp Dermatol. 2017; 26:659-67.

8. Abokwidir M, Fleischer AB. Additional evidence that rosacea pathogenesis may involve demodex: New information from the topical efficacy of ivermectin and praziquantel. Dermatol Online J. 2015;21(9):13030/ qt13v249f5.

9. Coston TO. Demodex folliculorum blepharitis. Trans Am Ophthalmol Soc. 1967;65:361-92.

10. López-Ponce D, Zuazo F, Cartes C, Salinas-Toro D, Pérez-Valenzuela C, Valenzuela F, et al. High prevalence of Demodex spp. infestation among patients with posterior blepharitis: Correlation with age and cylindrical dandruff. Arch Soc Esp Oftalmol. 2017;92(9):412-8.

11. Ayyildiz T, Sezgin FM. The effect of ocular Demodex colonization on Schirmer test and OSDI scores in newly diagnosed dry eye patients. Eye Contact Lens. 2020;46(Suppl 1):S39-S41.

12. Soler Ferrández FL. Demodex, manejo y tratamiento [Internet]. Elche, España: FacoElche; 07/03/2018 [consultado: mayo de 2021]. Disponible en: https://www.facoelche.com/demodex-manejo-tratamiento
13. Yam JC, Tang BS, Chan TM, Cheng AC. Ocular demodicidosis as a risk factor of adult recurrent chalazion. Eur J Ophthalmol. 2014;24:159-63.

14. Liang L, Ding X, Tseng SCG. High prevalence of Demodex brevis infestation in chalazia. Am J Ophthalmol. 2014;157:342-8.e1.

15. Cheng S, Zhang M, Chen H, Fan W, Huang Y. The correlation between the microstructure of meibomian glands and ocular Demodex infestation: A retrospective case-control study in a Chinese population. Medicine (Baltimore). 2019;98:e15595.

16. Kheirkhah A, Casas V, Li W, Raju VK, Tseng SC. Corneal manifestations of ocular demodex infestation. Am J Ophthalmol. 2007;143:743-9.

17. Liu J, Sheha H, Tseng SC. Pathogenic role of Demodex mites in blepharitis. Curr Opin Allergy Clin Immunol. 2010;10:505-10.

18. Luo X, Li J, Chen C, Tseng S, Liang L. Ocular demodicosis as a potential cause of ocular surface inflammation. Cornea. 2017;36:S9-S14.

19. Liang L, Liu Y, Ding X, Ke H, Chen C, Tseng SCG. Significant correlation between meibomian gland dysfunction and keratitis in young patients with Demodex brevis infestation. Br J Ophthalmol. 2018;102(8):1098-102.

20. Dougherty JM, McCulley JP. Bacterial lipases and chronic blepharitis. Invest Ophthalmol Vis Sci. 1986;27:486-91.

21. Suzuki T. Inflamed obstructive meibomian gland dysfunction causes ocular surface inflammation. Invest Ophthalmol Vis Sci. 2018;59(14):DES94DES101.

22. Patel NV, Mathur U, Gandhi A, Singh M. Demodex blepharo keratoconjunctivitis affecting young patients: A case series. Indian J Ophthalmol. 2020;68(5):745-9.

23. McCulley JP, Sciallis GF. Meibomian keratoconjunctivitis. Am J Ophthalmol. 1977;84:788-93.

24. Donmez O, Akova YA. Pediatric ocular acne rosacea: Clinical features and long term follow-up of sixteen cases. Ocul Immunol Inflamm. 2021;29(1):57-65

25. Gallo RL, Granstein RD, Kang S, Mannis M, Steinhoff M, Tan J, Thiboutot D. Standard classification and pathophysiology of rosacea: The 2017 update by the National Rosacea Society Expert Committee. J Am Acad Dermatol. 2018;78(1):148-55.

26. Forton F, de Maertelaer V. Papulopustular rosacea and rosacea-like demodicosis: two phenotypes of the same disease? J Eur Acad Dermatol Venereol. 2018;32(6):1011-6.

27. Deeks ED. Ivermectin: a review in rosacea. Am J Clin Dermatol. 2015; 16:447-52.

28. Brown M, Hernández-Martín A, Clement A, Colmenero I, Torrelo A. Severe demodexfolliculorum-associated oculocutaneous rosacea in a girl successfully treated with ivermectin. JAMA Dermatol. 2014;150(1):61-3.

29. Ebbelaar C, Venema AW, van Dijk MR. Topical ivermectin in the treatment of papulopustular rosacea: A systematic review of evidence and clinical guideline recomendations. Dermatol Ther. 2018;8:379-87.

30. Sahni DR, Feldman SR, Taylor SL. Ivermectin 1\% (CD5024) for the treatment of rosacea. Expert Opin Pharmacother. 2018;19(5):511-6.

31. Cardwell LA, Alinia H, Moradi Tuchayi S, Feldman SR. New developments in the treatment of rosacea - role of once-daily ivermectin cream. Clin Cosmet Investig Dermatol. 2016;9:71-7.

32. Sobolewska B, Doycheva D, Deuter CM, Schaller M, Zierhut M. Efficacy of topical ivermectin for the treatment of cutaneous and ocular rosacea. Ocul Immunol Inflamm. 2020 Apr 7:1-5. doi: 10.1080/09273948.2020.1727531. Online ahead of print.

33. Ávila MY, Martínez-Pulgarín DF, Rizo Madrid C. Topical ivermectin-metronidazole gel therapy in the treatment of blepharitis caused by Demodex spp.: A randomized clinical trial. Cont Lens Anterior Eye. 2021; 44(3):101326. 